\title{
Image processing of cylinder wake generation
}

\author{
J. Massons, Jna. Gavaldà, and F. Dlaz \\ Laboratori de Fisica Aplicada, Departamento Quimica, Universidad de Barcelona, P. Imperial Tarraco, I, \\ 43005 Tarragona, Catalunya, Spain \\ LI. Solé \\ Institut "Jaume Almera," CSIC, C/Martí Franqués, s/n, 08028 Barcelona, Catalunya, Spain
}

(Received 26 October 1987; accepted 14 February 1989)

\begin{abstract}
In the present study, image processing techniques are applied to the chronophotographic visualizations of a cylinder wake generation. The flow patterns obtained by means of tracer particles are digitalized and processed in order to characterize the flow. This characterization is carried out by determining the evolution of the geometric parameters governing the wake, together with the streamfunction, vorticity, and pressure distributions. The present study reaches the moment of shedding of the first pair of vortices.
\end{abstract}

\section{INTRODUCTION}

The study of the unsteady flow generated by an impulsively started cylinder, despite its simple geometry and the fact that it poses a classic problem in the field of fluid mechanics, is still a subject open to further research. This is due, particularly in the case of moderate and high Reynolds numbers, to the extraordinarily complex nature of the phenomena involved, which do not allow trivial interpretations.

The flow patterns that are obtained in the cylinder wake generation basically depend on both the Reynolds number and the moment of observation of the unsteady process. The study, undertaken by Coutanceau and Bouard, ${ }^{1}$ enables the existence of three differentiated flow regimes to be established: for low Reynolds numbers $(4.4 \leqslant \operatorname{Re} \leqslant 60)$, moderate ones $(60 \leqslant \operatorname{Re} \leqslant 500)$, and high ones ( $\operatorname{Re} \geqslant 500)$. There exist other factors that exert a secondary and disruptive influence on the above parameters. Included among these is the surface roughness of the cylinder ${ }^{2}$; its aspect ratio and the blockage relation ${ }^{3}$ must be pointed out.

The difficulty in the numerical integration of the Navier-Stokes equations for this geometry in an unsteady regime ${ }^{4-12}$ leads to the need for accurate experimental results in order for them to be correlated to the previous ones. This will, in turn, validate the hypotheses necessary for the numerical solution of the problem. Despite the importance of both this study and the interpretation of these types of flows for moderate and high Reynolds numbers, the experimental work carried out so far ${ }^{13-19}$ has, in most cases, been restricted to low or moderate Reynolds numbers and is always limited to the first phase of the process.

The aim of the present study is to characterize the generation of a cylinder wake for a high Reynolds number $(\operatorname{Re}=2000$ ). Thus both the first unsteady phase and a second phase are analyzed, the latter being characterized by the mutual interaction of the first pair of vortices formed, which leads to the self-destruction of the coherent vorticity. Image processing techniques are applied to the chronophotographic visualizations obtained, and the two-dimensional distribution of the different fluid-dynamic variabies.associated with the flow are evaluated. Image processing techniques were also applied by Dimonakis et al. ${ }^{20}$ and Kobayashi et $a l^{21,22}$ in the experimental study of mixing layers and wakes, respectively.

\section{EXPERIMENTAL DETAILS}

The visualizations were obtained in the towing water tank $\left(50 \mathrm{~cm} \times 30 \mathrm{~cm} \times 250 \mathrm{~cm}\right.$ ) described by Massons ${ }^{23}$ (Fig. 1). A guiderail system, on which a carriage moved, enabled the model, the lighting system, and the photographic equipment to be shifted, free of vibrations. The two-dimensionality of the flow was checked in order to elect the maximum diameter of the cylinder, which allows a two-dimensional flow to be maintained for a cylinder of $30 \mathrm{~cm}$ in length. As a result of this preliminary study a cylinder of $D=6 \mathrm{~cm}$ in diameter was chosen. The blockage ratio is 0.12 ; this value is lower than the one used by Honji and Taneda ${ }^{13}$ and Bouard and Coutanceau ${ }^{24}$ for similar Reynolds number. The cylinder was made of highly polished Plexiglas. Its roughness is less than $10^{-5}$ times the cylinder diameter. The illumination of the observation plane was achieved by means of two movie lights, each of $1000 \mathrm{~W}$, situated on both sides of the tank and collimated by $5 \mathrm{~mm}$ wide slits. The photographic recordings of the particle motions projected on the observation plane were obtained at regular time intervals using a Nikon FM2 camera, equipped with a Nikon MD-12 motor drive. The photographic film used for the visualizations was Ilford, having a $400 \mathrm{ASA}$. The time corresponding to each photograph had been obtained by calibration of the camera motor drive, and its evaluation adimensionalized with respect to the time taken by the potential-steady flow in covering a distance equal to the cylinder diameter; that is, $t^{*}=t U_{0} / D$, where $U_{0}$ is the free-stream velocity in the final steady regime and $D$ is the cylinder diameter. The exposure time for obtaining the photographs was $t_{\text {exp }}=\frac{1}{4} s\left(t_{\text {exp }}^{*}\right.$ $=0.137$ ), an interval in which it may be allowed that the velocity field of the flow does not evolve to any appreciable extent, while the camera shutter remains open. Synchronism between the start of movement and the taking of the photographs was achieved by using a Nikon MC-4 remote-control cable, which activates the camera motor drive simultaneously with the starting of the cylinder motion. An analysis of the time evolution of the length of the traces located in the potential zone of the flow is carried out in order to evaluate the time history of the translation velocity of the cylinder. This preliminary test has concluded that the velocity of the cylinder is constant after $t^{*}=0.074$ (first photograph). At this point the detachment of the boundary layer has not occurred 


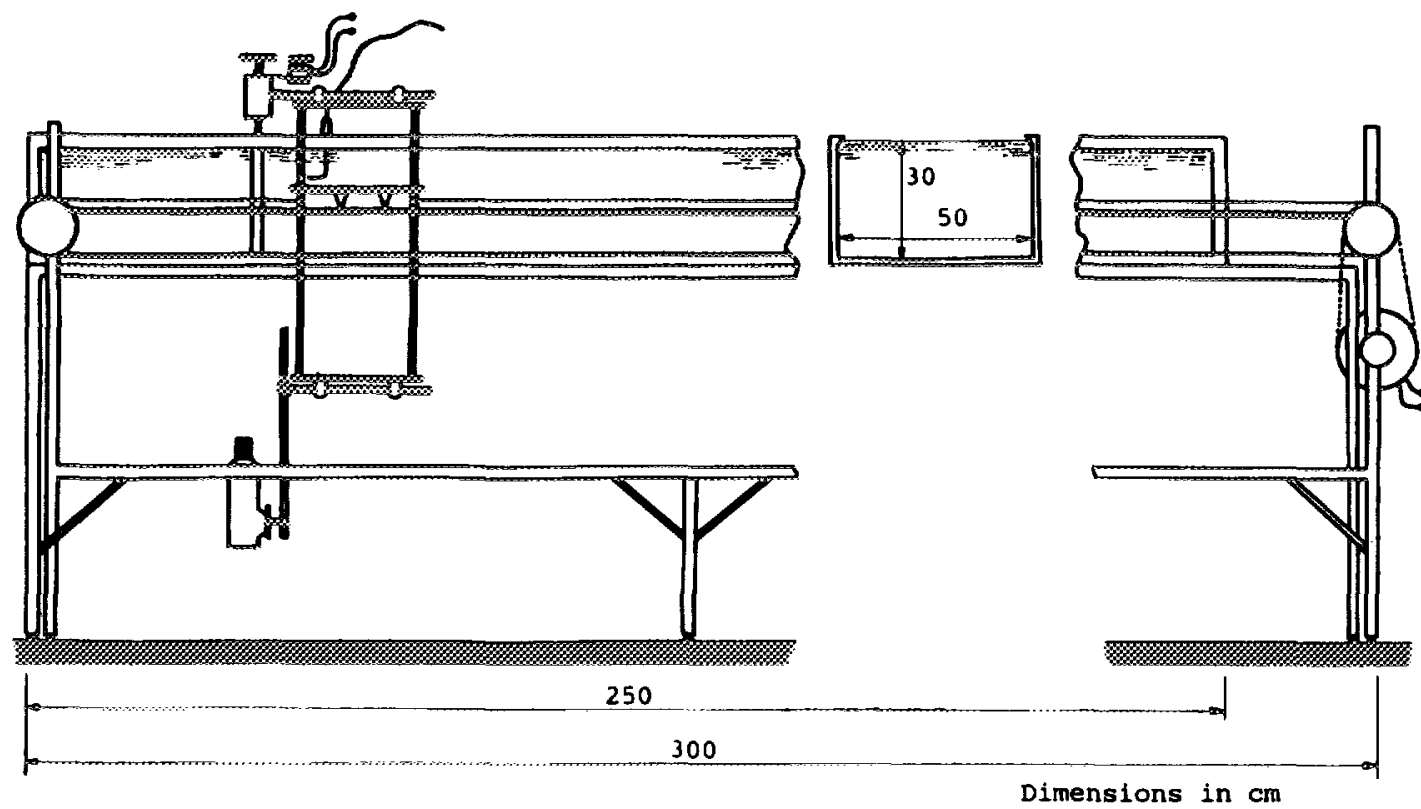

FIG. 1. Sketch of the flow visualization channel.

yet. Consequently, the starting of the cylinder can be considered almost instantaneous.

Magnesium powder (2-5 $\mu \mathrm{m}$ in diameter) was used to provide the tracer particles for the chronophotographic recordings. The particles were spread homogeneously throughout the flow. In order to obtain a stationary and passive distribution of particles it takes more than $30 \mathrm{~min}$ between the spreading of the particles and the moment of the flow visualization. The spreading density resulted from a compromise between a value high enough to give suitable accuracy in the later numerical calculations and low enough to enable the localization and individualization of each particle path in the applied image processing.

Figures 2 (a)-2(f) show the visualizations corresponding to the first stage of the flow, between the moments $t^{*}=1.11$ and $t^{*}=6.32$, after which the first pair of vortices formed in the wake have cancelled each other's vorticity, through mutual interaction. A photographic recording was taken every $t^{*}=0.347$, although only the results at time intervals of $t^{*}=1.041$ are presented in this paper.

\section{IMAGE PROCESSING PROCEDURES}

The photographic recordings containing the paths traced in the flow by the magnesium particles were digitalized with a Bosch monochromatic video camera. This camera enables a resolution of $512 \times 512$ pixels, with $256 \mathrm{grey}$ levels per pixel. The minimum distance to be resolved in the true flow scale was $D / 186$.

The digital processing of the images obtained was carried out with IBAS/IPS equipment. The first step in processing involved the homogenization of the lighting. This was achieved by subtracting from the original image the image resulting from applying a spatial low-pass filter with a low cutoff frequency to the former. In order to increase the contrast between the particle traces and the image background, a bandpass filter was applied, which simultaneously tended to heighten the contrast between the traces and soften their

outline. An example of the process used for isolating and localizing the traces is presented in Figs. 3(a) $-3(\mathrm{~g})$. Figure 3 (a) shows the image corresponding to the photograph presented in Fig. 2(d) reproduced on the display of the computer. Figure 3(b) presents the filtered image. Figures $3(a)$ and 3 (b) also include the transversal grey level profile between $Y / D=0.4$ and $Y / D=0.7$ at $X / D=2$. An increase in the

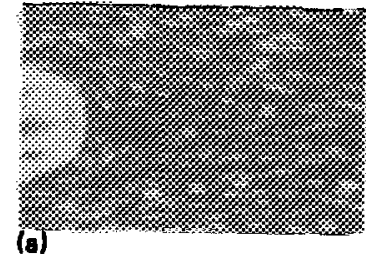

(8)

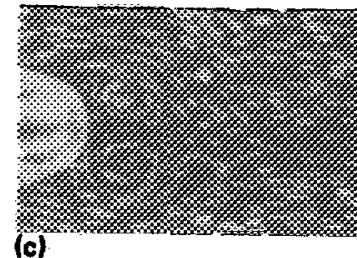

(c)

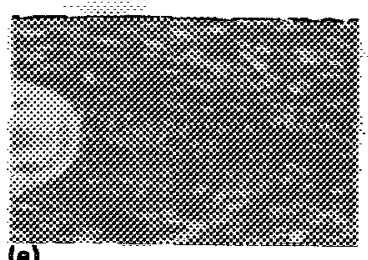

(a)

FIG. 2. Visualizations of the flow. (a) $t^{*}=1.115$; (b) $t^{*}=2.156$; (c) $t^{*}=3.197$; (d) $t^{*}=4.238$; (e) $t^{*}=5.279$; (f) $t^{*}=6.320$. 
at the same time, long enough to avoid the measurement error of particle paths. The most important disruptive effect produced by the velocity evolution during the exposure time is the generation of curved traces in some areas of the flow but, in the photographs analyzed in this study this effect has a relative importance only in the center of the vortices, giving not a significant contribution in other zones of the flow. Consequently the global error is coincidental with the measurement error, with the exception of the core of vortices, where the error increases because the nonlinear evolution of the velocity.

The measuring errors are related to the extracting and measuring processes and must be classified according to the different way of measure used in this work: automatic or manual. The high contrast existing between the traces and the background produces in the traces a relative insensitivity to the binarization process, when the location of the traces is automatic. Taking into account also the traces that must be manually measured, the error associated to the measuring process can be estimated as $8 \%$ in the stagnation zones and lower than this value in the rest.

After the traces were located and measured, the velocity field in the flow was determined. The evaluation of the velocity field from the distribution of the traces presupposed two things: that the tracer particles were of suitable physical characteristics to enable the flow evolutions in the observation plane to be followed accurately without modifying them (passive contaminant), and that the exposure time ( $t$ exp $=0.137$ ) used in the photographs was short enough to avoid important temporal evolutions being produced in the dynamic field in the interval of time recorded by the photograph. The velocity distribution in $\mathrm{cm} / \mathrm{sec}$ was obtained from the particle path distribution in pixels, taking as change variables the exposure time used and the cylinder diameter measured in pixels on the image and in centimeters in the true flow scale.

\section{NUMERICAL PROCEDURES}

The first step in the numerical procedure involved the velocity evaluation from the geometrical characteristics of the traces. Let $C X, C Y, l$, and $\alpha$ be the coordinates of the gravity center, length, and angle with $O X$ axis of a trace. The velocity components $u_{(C X, C Y)}$ and $v_{(C X, C n)}$ at the point $(C X, C Y)$ are obtained by

$$
\begin{aligned}
& u_{(C X, C n)}=l \cos (\alpha) / t_{\exp }, \\
& v_{(C X, C n)}=l \sin (\alpha) / t_{\exp },
\end{aligned}
$$

$t_{\text {exp }}$ being the exposure time of the photographs. The picture was recovered by a square grid. The evaluation of the velocity components $u(i, j), v(i, j)$ at the mesh point $(i, j)$ of the grid was carried out by linear adjust of the velocity values corresponding to the traces located inside a circle centered on the mesh point and with a radius equal to the mesh interval, as

$$
\begin{aligned}
u_{(C X, C \gamma)}= & u(i, j)+\left.\frac{\partial u}{\partial x}\right|_{(i, j)}(C X-i \Delta x) \\
& +\left.\frac{\partial u}{\partial y}\right|_{(i, j)}(C Y-j \Delta y),
\end{aligned}
$$

$$
\begin{aligned}
v_{(C X, C \gamma)}= & v(i, j)+\left.\frac{\partial v}{\partial x}\right|_{(i, j)}(C X-i \Delta x) \\
& +\left.\frac{\partial v}{\partial y}\right|_{(i, j)}(C Y-j \Delta y) .
\end{aligned}
$$

From this set of equations, $u(i, j)$ and $v(i, j)$ are determined by means of the least square method. If there are not enough traces inside the circle, or the correlation coefficient is lower than 0.85 , then the adjustment was effected from the eight traces nearest to the mesh point. The number of mesh points used to generate the velocity distribution was $21 \times 21$, which led to a mesh interval in the $X$ and $Y$, directions of approximately $\Delta x=\Delta y=D / 8$. This value was selected in such a way that, in the process of adjustment, the velocity value at each mesh point could be obtained in most cases from traces located at a distance not greater than a mesh interval.

The streamfunction distribution was computed by integration of the previously determined two-dimensional velocity field as

$$
\psi_{p}=\psi_{0}+\int_{0}^{p}(u d y-v d x)
$$

where $\psi_{0}$ is the streamfunction value at the origin of coordinates. The mesh point distribution of the streamfunction was calculated by means of the numerical integration of ( 3 ). Figure 4 shows the integral routes adopted. For the mesh points along the axes crossing at the coordinate origin, integration was made in either $X$ or $Y$ directions only, and for all the others the $X, Y$, and diagonal direction were arithmetically averaged. The streamfunction value at the origin of coordinates was assumed to be zero, and its value at another mesh point was computed by

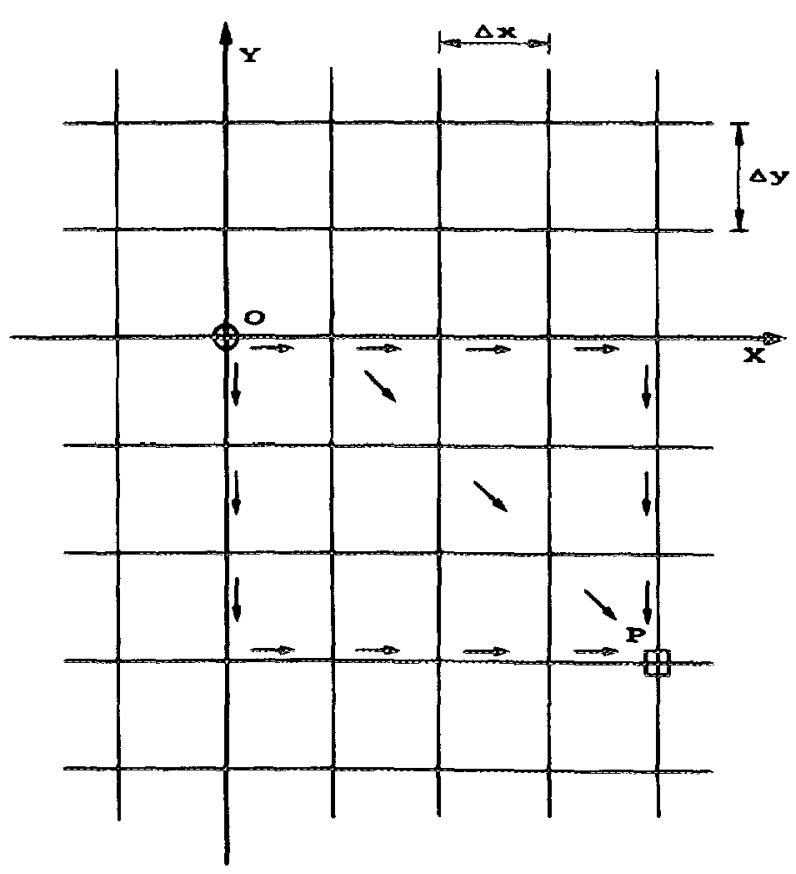

FIG. 4. Integral routes used for the streamfunction and pressure calculations. 


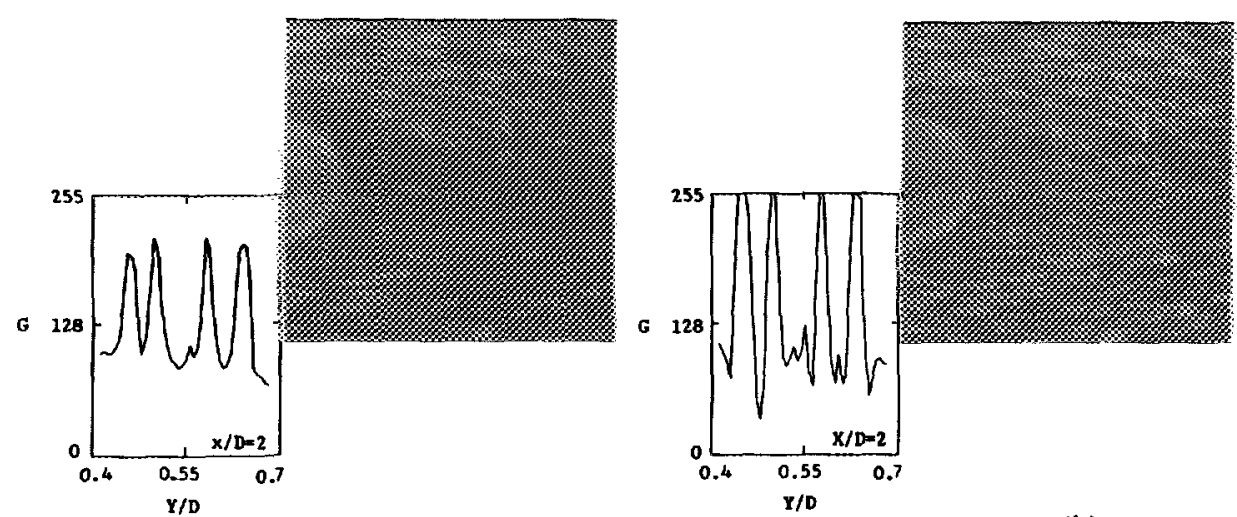

(a)

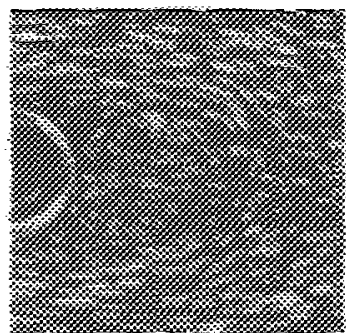

(d)

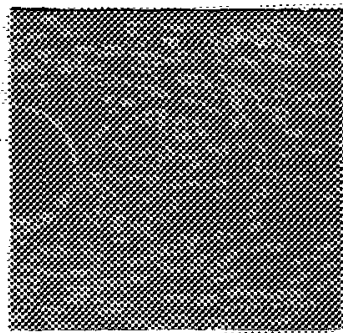

(e) (b)

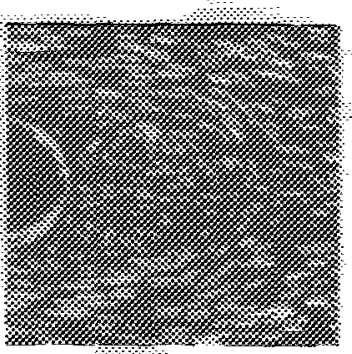

(f)

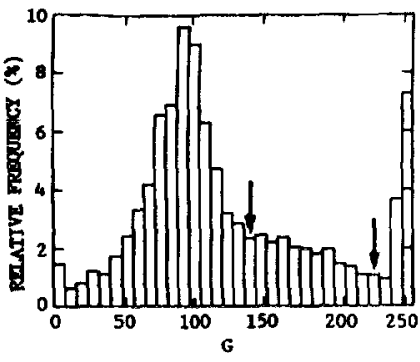

(c)

FIG. 3. An example of the trace identification process. (a) Original image; (b) filtered image; (c)grey level histogram of the filtered image; (d) binary image obtained for $G_{1}=136$; (e) binary image obtained for $G_{2}=225$; (f) binary image obtained from the common traces; ( $\mathrm{g}$ ) final binary image.

contrast between the traces and the background can be observed, which favors the detection process applied later. A suitable threshold level was chosen to distinguish between the traces and the background. This value was obtained from the analysis of the grey level histogram of the filtered image using Otsu's method. ${ }^{25}$ The threshold value for the histogram presented in Fig. 3 (c) is $G_{1}=136$. In this way, the grey level value of each pixel was used as a discriminant function, classifying each pixel as belonging to a trace or to the background. The binary image obtained for the abovementioned threshold level is shown in Fig. 3(d). As can be seen, at some times background noise has been incorrectly classified as a trace; consequently it is necessary to select a value greater that $G_{1}$ as a more adequate threshold. This value is chosen as $G_{2}=\left(G_{1}+3 \times 255\right) / 4=225$ in the present work. Figure $3(\mathrm{e})$ shows that no background noise is present in the image when the binarization process is effectuated with the new threshold. Unfortunately, a strong reduction of the length of some traces was produced. In order to improve the process, a new image [Fig. 3(f)] had been obtained from the first binarization [presented in Fig. 3(d)], subtracting the traces which were not simultaneously in both binarizations. This image is coincidental with the one obtained from the lower threshold level, but without the background noise. Finally, Fig. $3(\mathrm{~g})$ shows the distribution after manually eliminating the traces that present characteristics clearly uncorrelated with the neighboring ones (basi- cally the overlapped traces). The detected traces were visualized by superimposing them in overlay form on the original image. The traces that had not been located automatically, are determined manually. The proportion of automatically reconstructed traces is of about $68 \%$.

Once the image was in correct binary form, each of the particle paths (with pixels of grey level 255) surrounded by a background (grey level 0 ) was assimilated to an object. A measurement program carried out a screen scan locating each of these objects, individualizing them, and extracting the basic geometrical characteristics for determining the velocity field. The selected geometrical characteristics were the coordinates of the gravity center of the path, the maximum distance between two pixels of the trace, and the angle formed by this segment and the horizontal axis. The previous knowledge of the flow allows us to distinguish the initial position between both extremes of each trace. The detector used in locating the particle paths needed a suitable connection level to guarantee that the finer traces were not divided in the identification process.

There are some potential sources of error affecting the accuracy of the trace characterization process. From among these, the errors produced by the nonlinear behavior of the velocity field during the exposure time and the ones induced through the measurement process of the traces. The exposure time of the camera should be short enough to diminish the effect of nonlinear velocity evolution for its duration and, 


$$
\begin{aligned}
& \begin{array}{l}
\psi(i+\eta j \\
=
\end{array} \quad \psi(i, j)-\eta \Delta x[v(i, j)+v(i+\eta, j)] / 2 \\
& \quad+\xi \Delta y[u(i+\eta j)+u(i+\eta, j+\xi)] / 2,
\end{aligned}
$$

where, adopting suitable values for $\eta$ and $\xi$ parameters $(-1,0$, or 1$)$, allows us to obtain the streamfunction value at each mesh point if it is known in its neighbor. If one or more of these integral routes crosses the cylinder, its contribution was not considered in the average. In order to minimize and homogenize the accumulated integral errors, the coordinate origin was sequentially located at each mesh point. The final streamfunction distribution was obtained by averaging the streamfunction results obtained for each origin considered.
The vorticity distribution was obtained from that of the mesh point velocity. At a mesh point $(i, j)$, the vorticity $\omega(i, j)$ was obtained as

$$
\begin{aligned}
\omega(i, j)= & \frac{v(i+1, j)-v(i-1, j)}{2 \Delta x} \\
& -\frac{u(i, j+1)-u(i, j-1)}{2 \Delta y} .
\end{aligned}
$$

The pressure distribution was obtained from the unsteady Navier-Stokes equations in two-dimensional form and assuming incompressible flow.

For each $t$ * analyzed, the pressure gradients at the mesh point $(i, j)$ were computed as

$$
\begin{aligned}
\left.\frac{\partial p}{\partial x}\right|_{(i, j)}= & \rho\left(-u(i, j) \frac{u(i, j)-u(i-1, j)}{\Delta x}-v(i, j) \frac{u(i, j)-u(i, j-1)}{\Delta y}+v \frac{u(i+1, j)+u(i-1, j)-2 u(i, j)}{(\Delta x)^{2}}\right. \\
& \left.+v \frac{u(i, j+1)+u(i, j-1)-2 u(i, j)}{(\Delta y)^{2}}-\frac{u(i, j)-u_{-}(i, j)}{\Delta t}\right), \\
\left.\frac{\partial p}{\partial y}\right|_{(i, j)}= & \rho\left(-u(i, j) \frac{v(i, j)-v(i-1, j)}{\Delta x}-v(i, j) \frac{v(i, j)-v(i, j-1)}{\Delta y}+v \frac{v(i+1, j)+v(i-1, j)-2 v(i, j)}{(\Delta x)^{2}}\right. \\
& \left.+v \frac{v(i, j+1)+v(i, j-1)-2 v(i, j)}{(\Delta y)^{2}}-\frac{v(i, j)-v-(i, j)}{\Delta t}\right) .
\end{aligned}
$$

The suffix $(-)$ indicates that the velocity components correspond to the photograph taken just before $\left(t *-\Delta t U_{0}\right.$ $D$, where $\Delta t=0.631 \mathrm{sec}$ ). The integration of the above mentioned pressure gradients is obtained as in the streamfunction calculation case.

The calculation method involved here prevented determination of the vorticity and pressure distributions on the cylinder wall and on the edges of the image.

\section{RESULTS AND DISCUSSION}

Figure 5 shows a definition sketch of the geometric parameters used in characterizing the time evolution of the recirculating zone typical in this flow: downstream wake length, lateral separation between vorticity centers, longitudinal position of these centers, and maximum wake width.

In order to reach a better understanding of the mechanisms governing the genesis and evolution of the vortices, the two-dimensional distribution of the streamfunction, vorticity, and pressure was then obtained. Only the distributions corresponding to Figs. 2(b)-2(e) are shown in this paper, as they are the most representative of the unsteady flow considered here.

Figure 6 shows the histograms of the trace lengths detected in the different images analyzed, also indicating the corresponding spatial average and rms values of the distributions. It can be observed that, as the vortices evolve, the spatial average velocity in the observation window remains practically invariable, while the rms value increases progressively as a result of the wider range of velocities in different points of the flow. This proves that the flow undergoes an inevitable evolution toward increasing smaller scales.
In Fig. 7 the results obtained for the streamfunction, corresponding to Figs. 2(b)-2(e), may be observed. The results have been adimensionalized according to the following formula $\left[\psi^{*}=\psi /\left(U_{0} D\right)\right]$. A scale displacement was carried out for the values in order to obtain a streamvalue of 0 in the cylinder surface. The analysis of this figure reveals that the results are in agreement with those obtained from manual measurements by other authors ${ }^{24}$ for the evolution of the characteristic geometric parameters of the wake. The agreement of the shape obtained with the shape suggested by the particle path distribution may be observed. The streamfunction gradient in the two vortices is of opposite sign, since they turn in opposite directions. The symmetric nature of the wake evolution is maintained until the moment of shedding of the first pair of vortices, with the mutual canceling of their vorticity.

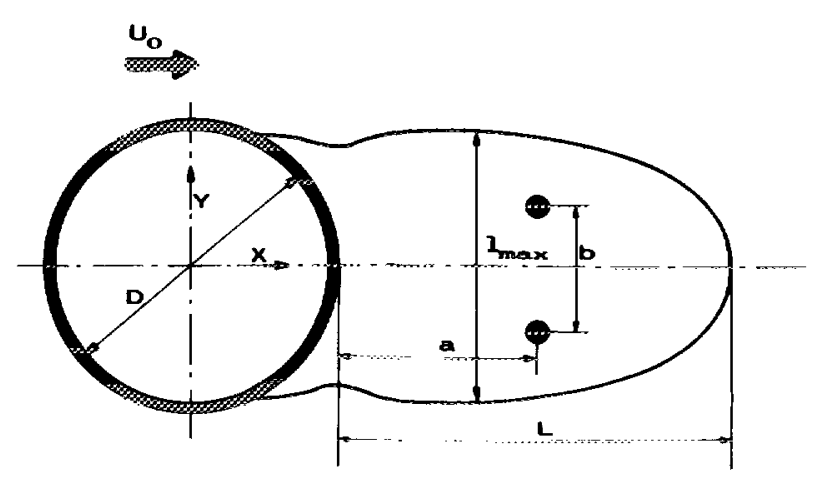

FIG. 5. Definition sketch of the geometrical parameters analyzed. 


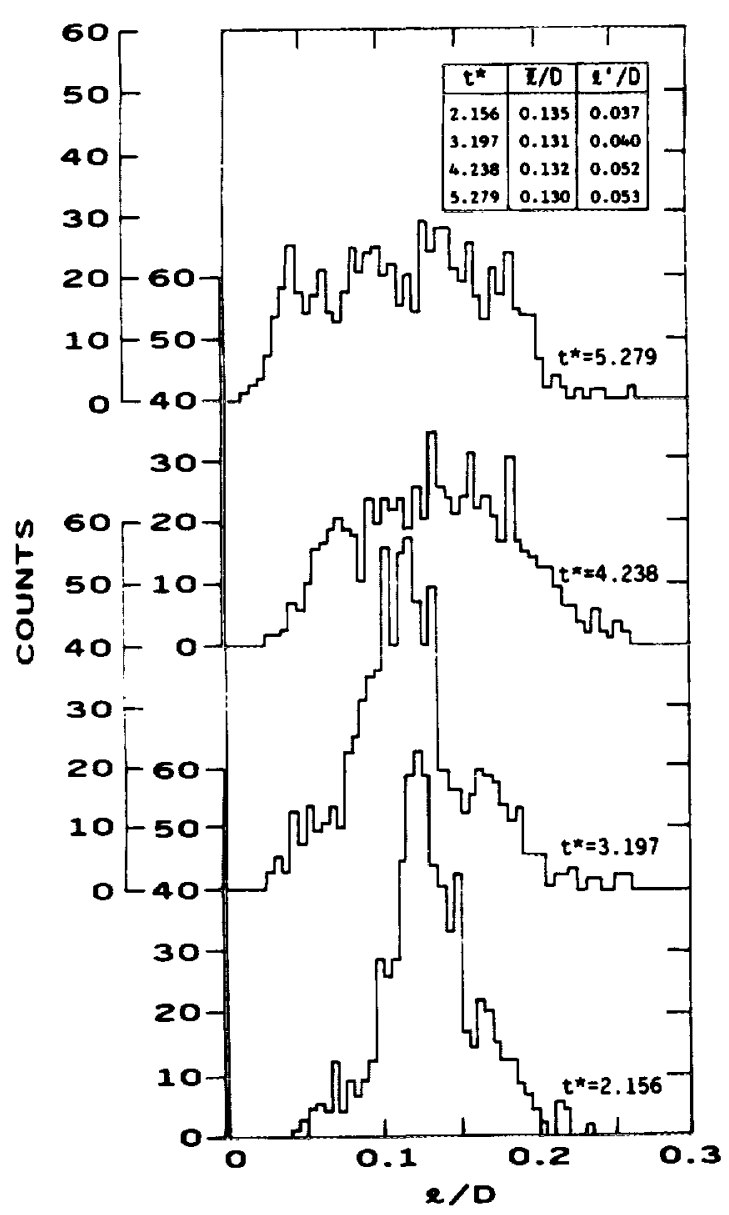

FIG. 6. Time evolution of the path lengths.

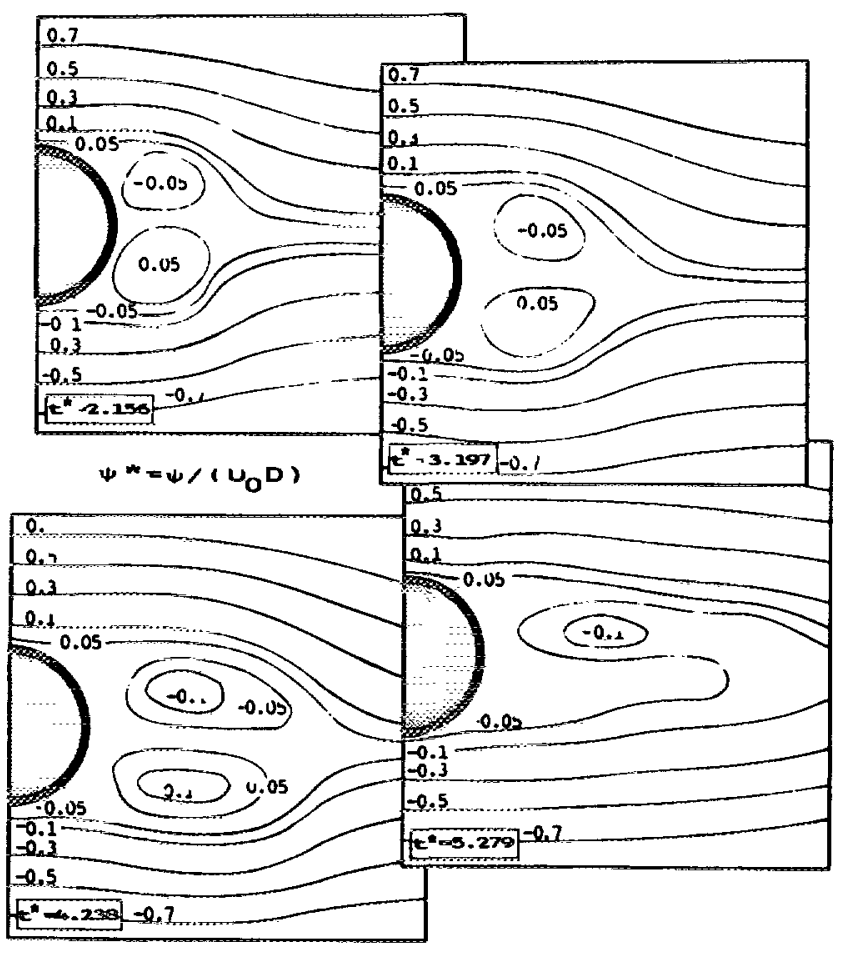

FIG. 7. Distribution of the streamfunction.

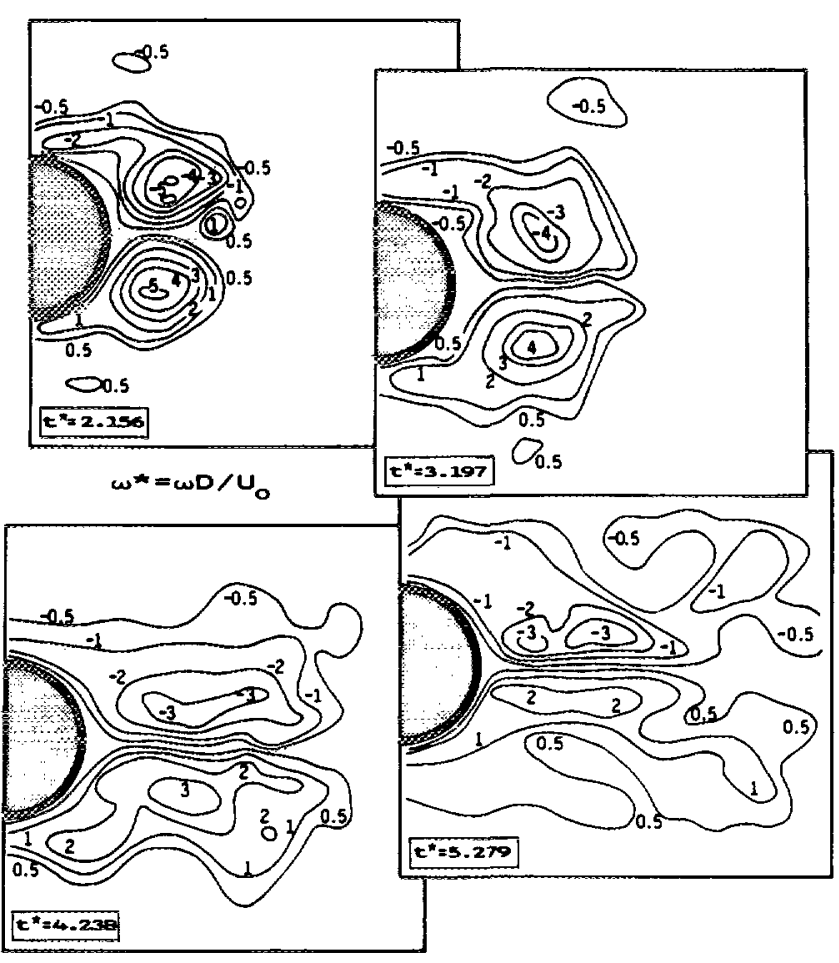

FIG. 8. Distribution of vorticity.

Figure 8 shows the vorticity distribution adimensionalized as $\omega^{*}=\omega D / U_{0}$. Two large coherent vorticity structures of opposite signs may be seen, corresponding to the first pair of counter-rotating vortices that are formed. The later process of vorticity diffusion, with the resulting drop in its maximum value as the vortices evolve and become separated from the cylinder, is particularly clear. This process accelerates for $t^{*}>3.5$. A considerable anisotropy of the vortical diffusion process may be observed. The lateral vorticity gradient evolves much more slowly and more uniformly than the longitudinal gradient. The existence of a dominant direction in the diffusion process is imposed by the flow itself, as it carries the generated vorticity downstream, thus favoring the diffusion. The high degree of symmetry observed in the vortical distribution is a consequence of the angular momentum conservation. This symmetry of the process remains until the moment of vortex destruction through their mutual interaction. Once the first pair of vortices shed themselves there follows a stage of chaotic movement, as a result of the recombination of their vorticity. This chaotic behavior of the flow favors the posterior setting up of the pseudostationary wake characterized by the emission of alternative Kármán vortices.

Figure 9 shows the two-dimensional pressure distributions. The pressure has been adimensionalized as $p^{*}=p /\left(\rho U_{0}^{2}\right)$, where $\rho$ is the fluid density. For this representation, the chosen criterion was that the adimensionalized pressure value in the upper left-hand corner of the image should be equal to 1 since it is a point that lies permanently beyond the influence of the cylinder and will therefore maintain a constant pressure value in the vortex evolution process. The presence of a maximum value of the pressure may be observed in the area of the downstream 


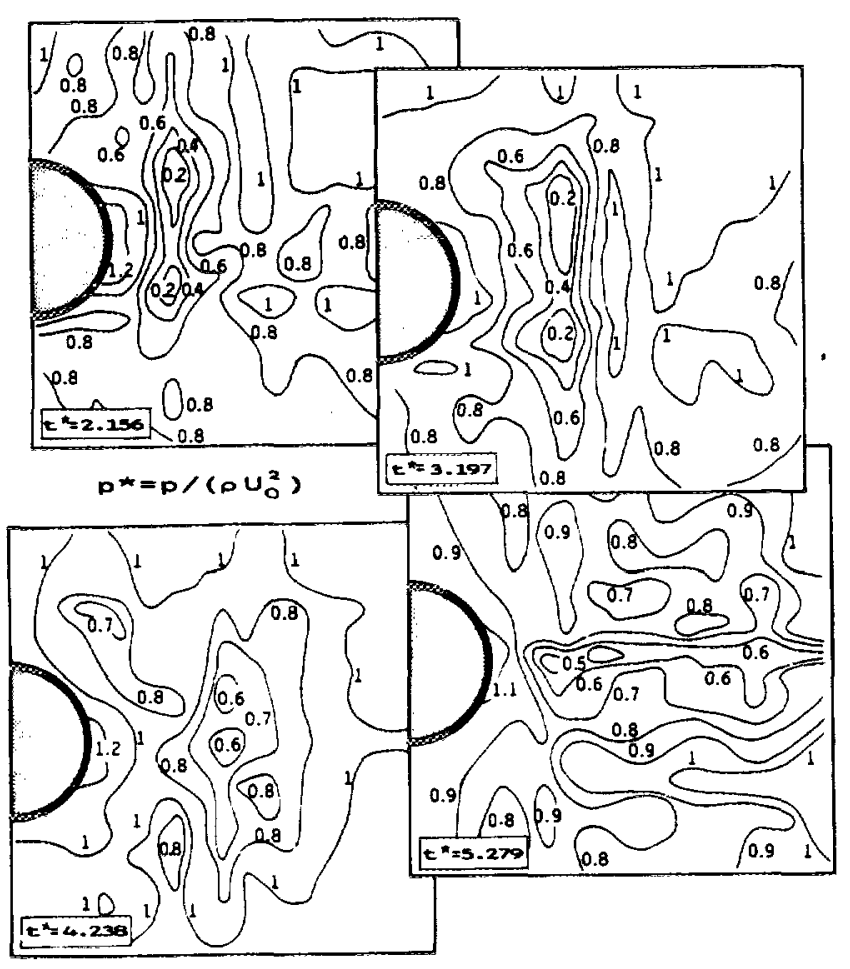

FIG. 9. Distribution of pressure.

stagnation point of the cylinder, which gives a practically constant value throughout the generation process of the wake. As can be seen, there exists a low-pressure zone with high gradients in the zone of intense vortical activity, and a more uniform distribution in the areas far away from the vortex centers. As in the case of the vorticity, this low-pressure zone decreases as the vortices evolve. This gradient is especially sharp for $t^{*}<3.5$. During the first stage of vortex evolution, the longitudinal pressure gradient is greater than the lateral gradient. In later stages of evolution this behavior is reversed, although a progressive homogenization of the pressure field can be observed as a consequence of the decrease in vortical activity and the homogenization of the flow. The evaluation of the convective and diffusive terms of the Navier-Stokes equation shows that the convective pressure contribution is notably higher than the diffusive contribution, thus proving that the transport of the dynamic variables is mainly carried out convectively, through the dynamic flow field.

Two types of errors affect the accuracy of the evaluation of the above-mentioned functions: errors related to the measurement process and those associated with the calculation method. The measuring procedure of the traces implies an error of $8 \%$, as discussed previously. Imaichi and $\mathrm{Ohmi}^{19}$ evaluated the accuracy of these numerical calculations and concluded that only in the mesh points located near the cylinder do the adjust techniques used become relatively inaccurate, and that the errors associated with the streamfunction, vorticity, and pressure distributions are, on average, approximately one-half of those obtained in the measuring process of the traces. Consequently, it can be established that the errors in the present distributions are, on average, lower than $4 \%$.

Figure 10 presents the evolution of the geometrical pa-

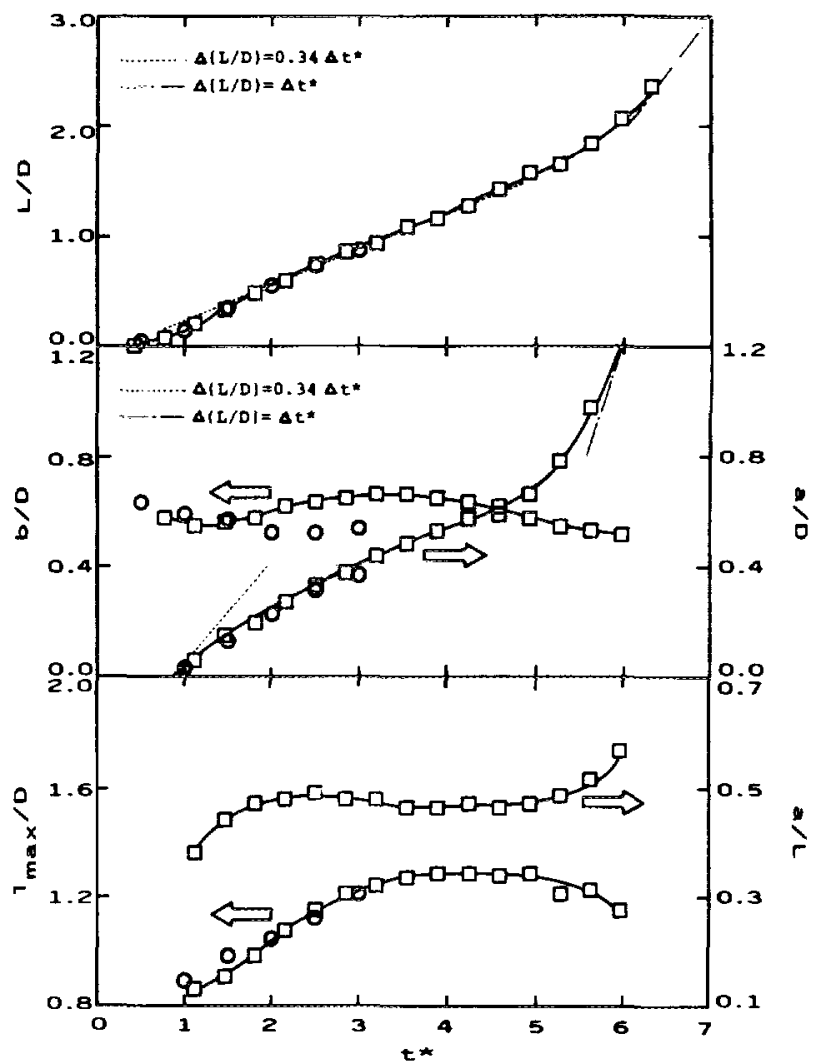

FIG. 10. Time evolution of the geometrical parameters of the wake ( $\square$ : present results; $O$ : Bouard and Coutanceau ${ }^{24}$ ).

rameters defined in Fig. 5, with respect to the adimensionalized time $t^{*}$. The downstream wake length $L$ and the maximum wake width $l_{\max }$ have been obtained from the streamfunction distribution (from the more external closed isoline) and the lateral separation, $b$, and longitudinal position, $a$, of the vorticity centers, were obtained from the vorticity distribution.

It may be seen that the length of the wake shows a tendency toward linear growth in stretches, with two inflection points located at $t^{*}=1$ and 5.5. The presence of the first inflection point was also observed by Bouard and Coutanceau $^{24}$ for $\operatorname{Re}=3000$. This inflection point corresponds to the moment when the flow changes its structure. This occurs with the settling of the first pair of vortices generated by the unstable whirling structures, which arise in parietal zones immediately after the start of motion of the cylinder. The linear behavior of the growth, which took place later, follows the law of Taneda. ${ }^{14}$ The second point of inflection appears right after shedding, at $t^{*}=5.5$. This may be explained by the fact that, just before shedding, a deceleration in the wake growth rate took place through the recombining of the vorticity of the two vortices and, after shedding, the chaotic behavior of the flow favors the downstream transport of the dynamic field of the wake, and produces a significant increasing in the growth of the wake length, which tends to fill the whole nonpotential flow area. In the stage before vortex shedding the momentum transport, downstream of the cylinder, is confined by the high coherent vorticity of the first two vortices generated by a strong pressure gradient at the beginning of motion.

The lateral separation between vorticity centers, $b / D$, 
shows a significant decreasing evolution in the first phase of the generation of the wake. This is due to the fact that the vortices are generated on both sides of the cylinder at diametrically opposite points, and then evolve rapidly toward the wake axis. For $t^{*}=1$, coinciding with the moment at which the first flow transition is produced, this parameter is stabilized at $b / D=0.6$. This value is maintained until the moment of shedding, when it drops slightly as a result of the approximation process undergone by the interacting vortices.

The time evolution of the longitudinal position of the vorticity centers, $a / D$, reveals behavior similar to that detected for $L / D$. The plot of the evolution of $a / L$ enables the minor differences between the evolution of these two parameters to be observed. During the first phase of the process, the downstream length of the closed wake is seen to evolve faster than the longitudinal position of the vortex centers. This results from the fact that the vortices are undergoing a generation phase and their vorticity centers are therefore more linked to the generating cylinder than the rest of the wake. For $t^{*} \geqslant 2$, the velocity of the vorticity centers is similar to that of the downstream front of the recirculating zone, reaching a practically constant value of $a / L=0.4$ until the moment of shedding, after which the velocity of the vorticity centers becomes greater than that of the front of the wake.

The maximum wake width increases in the first stage of the process. After $t^{*}=3.5$, its evolution is stabilized at $l_{\max } / D=1.2$. As the vortices shed from the cylinder, this parameter decreases as a result of the redistribution of vorticity, which enhances the confining action of the potential dynamic field. Comparing the evolution of $l_{\max } / D$ with that observed for $b / D$, it is seen that after the first stage of the process, in which the vorticity centers lie in the outer part of the recirculation zone, these centers gradually draw nearer to the wake axis, remaining in this situation until the moment of their shedding. Figure 10 also shows that the present results are consistent with those obtained by Bouard and Coutanceau ${ }^{24}$ for $\operatorname{Re}=3000$ for $t^{*}<3$.

Figure 11 shows the evolution of the velocity on the flow axis. These values are obtained from the lateral profiles of the longitudinal velocity. Results obtained by Bouard and Contanceau ${ }^{24}$ and Loc and Bouard ${ }^{10}$ for $\mathrm{Re}=3000$ are also included. A reasonable agreement between the present and previous results exists. The negative zone of the curves corresponds to the returning flow generated in the axis zone. It may be observed that when $t^{*}$ increases, the velocity of the returning flow increases for $t^{*}<3.5$, and decreases for $t *>3.5$. This behavior must be linked to the evolution of the vortices in two ways: first, a coherent growth of the vortices exists and second, the interaction between both vortices produces its destruction and the homogenization of the velocity field in the wake. The position of the second zero velocity point coincides with the closed wake length obtained from the streamfunction distributions (Fig. 10). Far away from the cylinder, the axial velocity shows an asymptotic tendency toward the free-stream velocity. This tendency becomes stronger as $t^{*}$ decreases.

The time evolution of the maximum velocity of the returning flow, $u_{\max }$, and its location, $d$, are plotted in Fig. 12. The results obtained by Bouard and Coutanceau ${ }^{24}$ are also included. The $u_{\max }$ results agree with the above-mentioned behavior of the vortices and show a decreasing tendency after $t^{*} \simeq 3.5$. The location of the maximum velocity, $d$, increases regularly with time. When the vorticity diffusion becomes relevant $\left(t^{*} \geqslant 4\right)$, the turbulent behavior of the wake causes a lack of definition in the results, especially for the ones involving $d / D$. This tolerance has been indicated in Fig. 12 by a shadowed area.

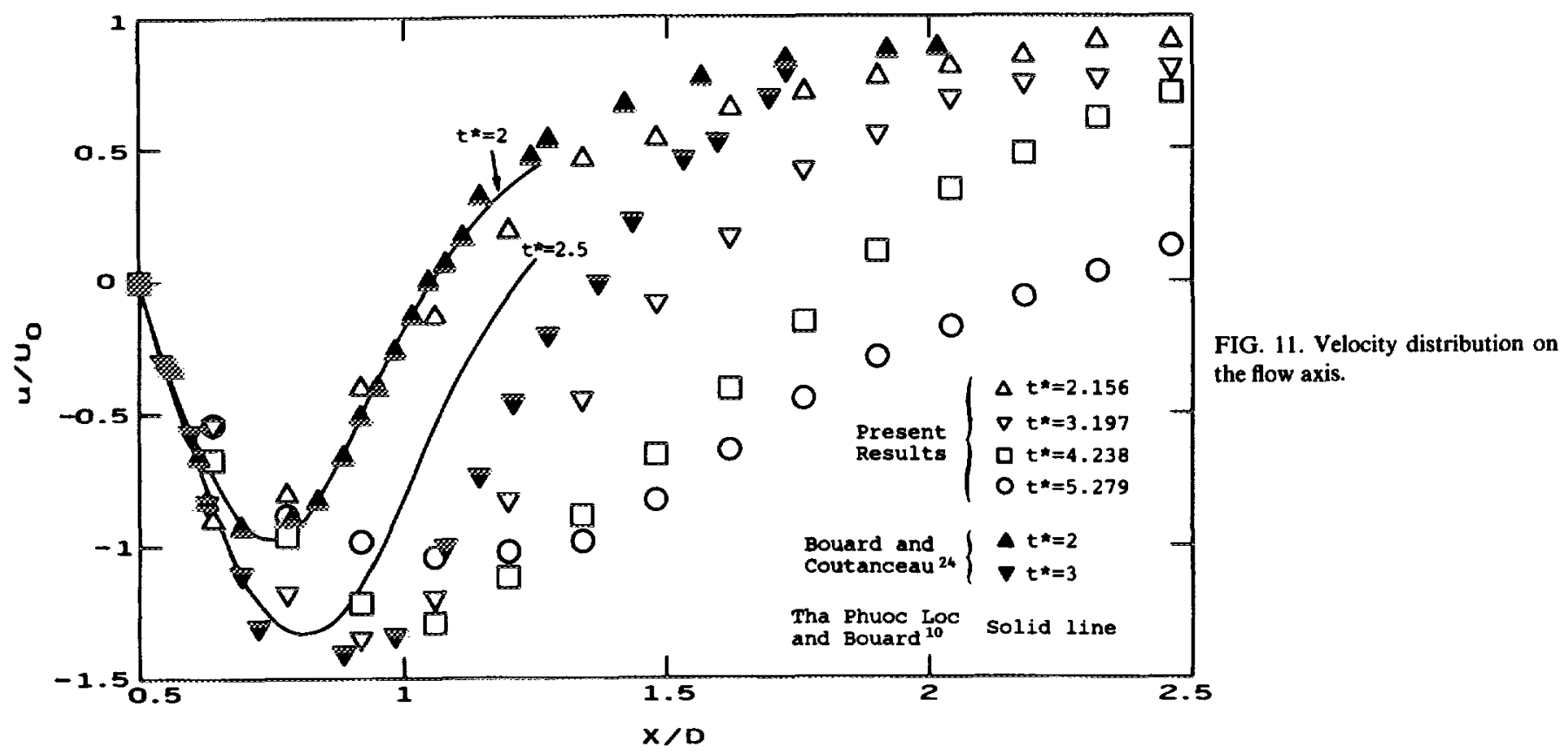




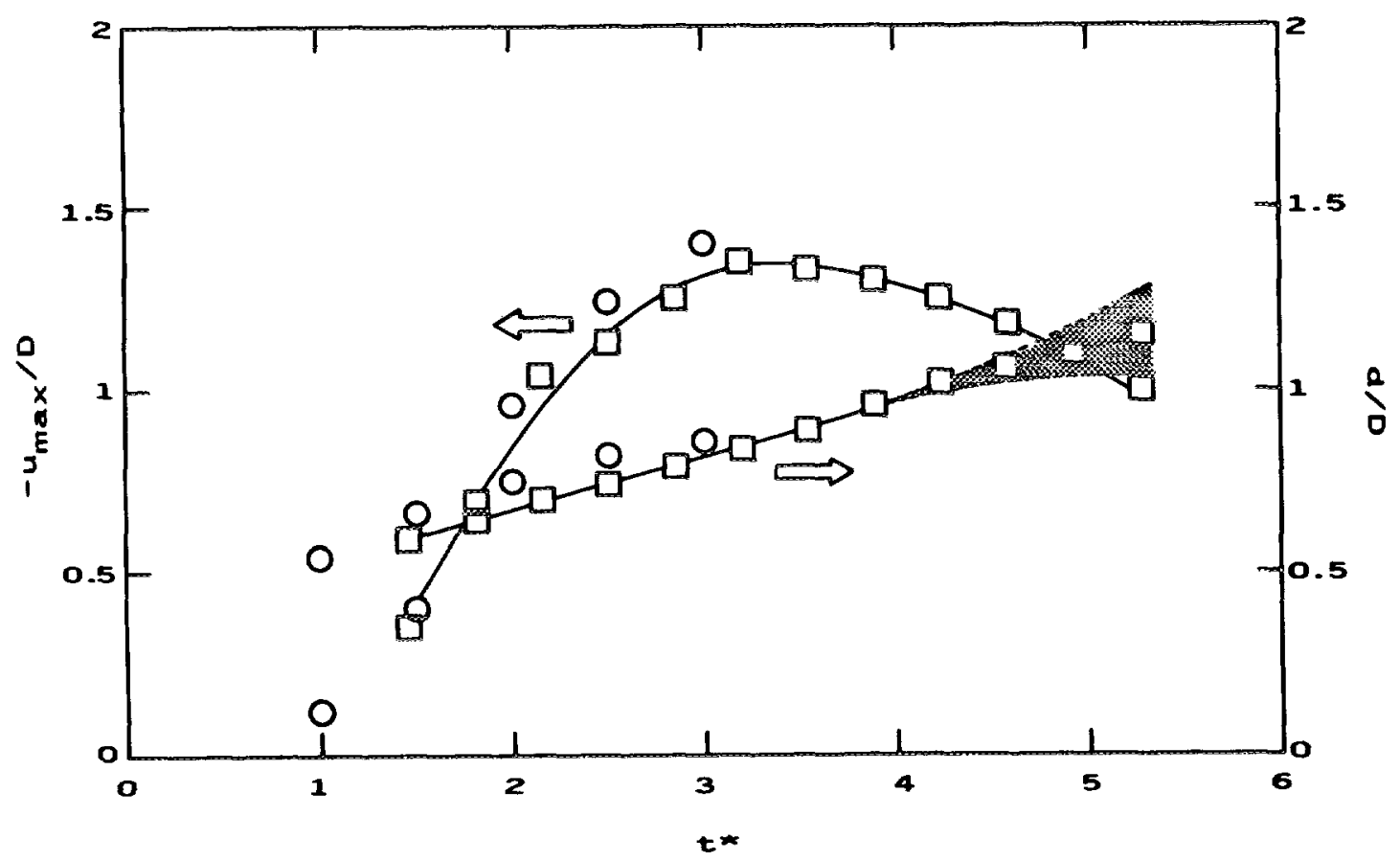

FIG. 12. Time evolution of the magnitude and of the location of the velocity maximum in the wake. ( $\square$ : present results; $O$ : Bouard and Coutanceau ${ }^{24}$.)

\section{CONCLUSIONS}

The image processing of the flow pictures obtained from two-dimensional flows constitutes a new way of analyzing the hydrodynamic field that has been established. The present results obtained in the unsteady flow generated by the implusively started cylinder both complete and complement other existing studies, although these studies have been based on more conventional techniques. The determination of the velocity field, carried out by means of image processing techniques, and the later determination of the fluid dynamic distributions (streamfunction, vorticity, and pressure) allow the physical interpretation of this complex flow generation and its time evolution. It is observed that, for $t^{*}<3.5$, the vortices evolve, maintaining high coherence and low lateral diffusion. An increase in the diffusion process is later observed and for $t^{*} \simeq 5.5$, the vortices shed. A flow with chaotic behavior is then generated, which favors the setting up of the pseudostationary wake and the alternative vortex shedding.

\section{ACKNOWLEDGMENTS}

The technical facilities provided by TAQSA and ALCUDIA S.A. are acknowledged.

The authors are also grateful for the financial support provided by CIRIT (Generalitat de Catalunya).
'M. Coutanceau and R. Bouard, C. R. Acad. Sci. Paris Ser. B 288, 45 (1979).

${ }^{2}$ A. Achenbach and E. Heinecke, J. Fluid Mech. 109, 239 (1981).

${ }^{3}$ G. S. West and C. J. Apelt, J. Fluid Mech. 114, 361 (1982).

${ }^{4}$ W. M. Collins and S. C. R. Dennis, J. Fluid Mech. 60, 105 (1973).

${ }^{5}$ H. Bar-Lev and H. T. Yang, J. Fluid Mech. 72, 625 (1975).

'F. D. Deffenbaugh and F. J. Marshall, AIAA J. 14, 908 (1976).

'V. A. Patel, Comp. Fluids 9, 435 (1981).

${ }^{8}$ Ta Phuoc Loc, J. Fluid Mech. 100, 111 (1980).

${ }^{9}$ M. C. Ece, J. D. A. Walker, and T. L. Doliganski, Phys. Fluids 27, 1077 (1984).

${ }^{10}$ Ta Phuoc Loc and R. Bouard, J. Fluid Mech. 160, 93 (1985).

"H. Badr and S. C. R. Dennis, J. Fluid Mech. 158, 447 (1985)

${ }^{12}$ A. Giorgini and J. R. Pravia, Rep. No. CE-HSE-85-04, Purdue University, 1985 .

${ }^{13}$ H. Honji and S. Taneda, J. Phys. Soc. Jpn. 27, 1668 (1969).

${ }^{14} S$. Taneda, Prog. Aerosp. Sci. 17, 287 (1977).

${ }^{15}$ M. Coutanceau and R. Bouard, J. Fluid Mech. 79, 231 (1977).

${ }^{16}$ M. Coutanceau and R. Bouard, J. Fluid Mech. 79, 257 (1977).

${ }^{17}$ A. E. Perry, M. S. Chong, and T. T. Lim, J. Fluid Mech. 116, 77 (1982).

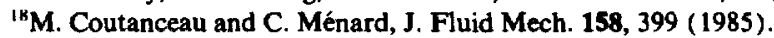

${ }^{19}$ K. Imaichi and K. Ohmi, J. Fluid Mech. 129, 283 (1983).

${ }^{20}$ P. E. Dimonakis, F. D. Debussy, and M. M. Koochesfahani, Phys. Fluids 24, 995 (1981).

${ }^{2 '}$ T. Kobayashi, Y. Yoshitake, T. Saga, and S. Segawa, International Sympasium on Physical and Numerical Flow Visualization, edited by M. L. Millet, J. H. Kim, and T. R. Heidrick (ASME, New York, 1985), Vol. 22, p. 39.

${ }^{22}$ T. Kobayashi, T. Saga, and S. Segawa, in Flow Visualization IV edited by C. Veret (Hemisphere, New York, 1987).

${ }^{23} \mathrm{~J}$. Massons, Thesis, Universidad de Barcelona, 1987.

${ }^{24}$ R. Bouard and M. Coutanceau, J. Fluid Mech. 101, 583 (1980).

${ }^{25}$ N. Otsu, IEEE Trans. Syst. Man Cybern. SMC-9, 62 (1979). 